\title{
Dialogue sur le handicap : réciprocité et altérité
}

\section{A Dialog on disability : reciprocity and otherness}

\section{Pierre ANCET ${ }^{1}$ \\ Marcel NUSS ${ }^{2}$}

\section{Résumé :}

Ce dialogue n'est pas celui d'un maître de conférence en philosophie (Pierre Ancet) interrogeant une personne en situation de dépendance physique (Marcel Nuss), mais celui de deux auteurs à part entière, où l'atteinte organique ne constitue qu'une spécificité d'expérience qu'il s'agit de décrire sans la pathologiser. Le handicap est envisagé dans sa dimension organique, psychologique et sociale, en tant que contrainte incapacitante mais aussi comme potentiel vecteur de découverte de capacités non-standard. Parmi celles-ci, les auteurs insistent sur la conscience du temps et la conscience du corps, d'autant plus inattendue ici qu'elle est thématisée par un homme entièrement paralysé, ce qui n'empêche ni la sensation fine ni la mobilité psychique et la puissance de réflexion, dont ce texte est un reflet.

\section{Summary :}

Mots-clés :

Handicap, capacités, philosophie, phénoménologie, conscience du corps, corps vécu, temps vécu

Key Words :

Disabilities, abilities, philosophy, phenomenology, body consciousness, lived body, lived time

La co-écriture de nos textes s'inscrit dans une relation de réciprocité et d'amitié : il ne s'agit pas de l'enquête d'un enseignant-chercheur interrogeant un écrivain vivant au quotidien avec un handicap, mais de la rencontre de deux hommes susceptibles d'échanger à propos du vécu du corps, au vécu du temps, du rapport à l'apparence, à la séduction, aux femmes, à la sexualité... Si un chercheur académique peut rester attaché à un certain degré de généralité dans ses développements, en revanche il ne saurait se prétendre ici expert, sinon de sa propre vie. Les concepts philosophiques employés ont pour but de conduire à une appréhension plus fine du vécu et à des interrogations originales, dont nous avons rendu compte dans différents articles et un livre ${ }^{3}$.

Le recours à la philosophie, selon un angle que l'on peut dire phénoménologique, suppose que l'exigence descriptive s'applique potentiellement à chacun des auteurs et que les concepts utilisés puissent être rendus suffisamment accessibles pour que chacun se les approprie. Celui de nous deux qui peut bouger son corps se trouve tout autant que l'autre dans l'obligation de décrire son expérience : ce qu'est la sensation de la verticalité d'un corps, de son ancrage au sol par les pieds,

\footnotetext{
1 Maître de conférences en Philosophie, responsable de l'axe "Soins, vie et vulnérabilité " au Centre Georges Chevrier, UMR 7366, CNRS - Université de Bourgogne.

${ }^{2}$ Ecrivain, consultant, formateur et conférencier

${ }^{3}$ Pierre ANCET et Marcel NUSS, Dialogue sur le handicap et l'altérité. Ressemblances dans la différence, Paris Dunod, 2012.
} 
de sa possibilité d'action physique, etc. S'il convoque pour ce faire la notion de corps vécu, il lui faudra la rendre accessible : ce «corps » n'est pas réductible à l'organisme et à ses conséquences fonctionnelles, mais englobe les sensations, émotions et représentations associées. Ce corps vécu n'a pas nécessairement de forme ou d'apparence, et ne se limite pas aux capacités objectives : on peut rêver de mouvement, de puissances corporelles dont on ne dispose pas dans le monde ordinaire. Il s'agit du corps tel qu'il est ressenti, dans une dimension psycho-somatique.

L'écart entre les perceptions et les représentations que nous aurions eu tous deux tendance à projeter sur le corps de l'autre était ici sensible. Dans la mesure où l'un de nous se trouve être totalement paralysé, à l'exception de son visage, on pouvait penser qu'il ne ressentait pas son corps immobile, ce qui est totalement faux ; qu'il ne pouvait se projeter dans le mouvement d'autrui pour le ressentir comme de l'intérieur, ce qui est également faux. Il nous a fallu travailler par hypothèses, essais et erreurs, pour confirmer ou infirmer des intuitions plus ou moins correctes concernant l'expérience de l'autre.

\section{Ces présupposés de méthode étant posés, commençons notre dialogue :}

Pierre : Je souhaiterais comme nous en avons l'habitude insister sur les capacités de chacun d'entre nous au lieu de n'insister que sur les incapacités (réelles ou supposées) que l'on projette sur autrui, notamment sur un corps visibilement atteint par le handicap. Afin de lutter contre un certain nombre de représentations discriminantes, je te propose d'insister essentiellement sur tes propres aptitudes pour contrer les représentations courantes de ce que peut un homme paralysé, vivant avec un handicap jugé «lourd». Si tes aptitudes ne sont pas directement liées à ton handicap physique, celui-ci t'a certainement permis de développer davantage certaines capacités, notamment me semble-t-il le contact visuel, l'accueil d'autrui et la présence à l'autre.

Marcel : D'emblée, il me semble primordial de mettre le handicap à sa juste place, il ne faut ni l'idéaliser, ni le stigmatiser. Évidemment, avoir un handicap, contraint la personne " handicapée » $\grave{a}$ " s'arranger » avec une spécificité plus ou moins intransigeante et dure à vivre mais, pour autant, ce n'est pas une fatalité, ce n'est pas une condition rédhibitoire. Les regards posés sur un être sont en général bien plus handicapants que le handicap lui-même; le regard social est surhandicapant, de ce fait, il pénalise la personne en situation de handicap en préjugeant, consciemment ou non, de ses potentialités. À l'instar de tout un chacun, je me suis construit à partir de compétences et de capacités particulières innées que j'ai fait le choix de faire fructifier. Indéniablement, le handicap a eu une influence sur mon parcours et mes choix de vie. À ce titre, si l'on prend l'exemple de Milton Erickson ${ }^{4}$, on peut se demander s'il aurait été qui il a été et fait ses découvertes majeures sans son handicap. En fait, à bien y réfléchir, ce n'est pas tant le handicap que l'emprisonnement (corporel, sensoriel, psychique) qu'il induit qui conduit certaines individualités à faire un travail de dépassement de soi dans les limites « carcérales » qui leur ont été imposées, donc dans le champ de leurs restrictions spécifiques de liberté ; par exemple, Primo Levi ou Nelson Mandela ont fait un tel travail de dépassement spirituel. Et ce constat soulève une question non négligeable : pourquoi une minorité d'humains, lorsqu'ils sont confrontés à une situation et à des conditions d'existence extrêmes, déploient-ils des ressources insoupçonnées d'adaptation libératrice et transcendante, quand la majorité s'étiole ou s'effondre? Pourquoi certaines personnes sont-elles résilientes et d'autres pas? À une époque, je me demandais pourquoi moi j'avais ces capacités et pas mes camarades. Être résilient peut être culpabilisant...

En tout cas, du fait de mon handicap, j'ai développé certaines qualités : acuité visuelle et auditive, empathie, développement intellectuel et créatif, parce qu'elles donnent du sens à ma vie, mettent en lien et créent du lien. Sans ces qualités, il me semble difficile d'être véritablement présent à l'autre, donc à soi.

\footnotetext{
${ }^{4}$ Milton Erickson, fondateur de l'hypnose éricksonienne, était atteint de poliomyélite. Il était par ailleurs daltonien, dyslexique et amusique, ce qui l'a très jeune conduit à s'intéresser aux différences individuelles de perception du monde.
} 
— La notion de présence à l'autre, telle que tu l'as conceptualisée ${ }^{5}$ et telle que tu la manifestes au quotidien, rejoint ce que Richard Shusterman ${ }^{6}$ appelle la conscience $d u$ corps dans ses conséquences éthiques. Elle permet de développer ses aptitudes sensorielles mais aussi morales en s'appliquant à percevoir en soi des sentiments vagues et désagréables d'hostilité que la bonne conscience refuse d'apercevoir (par exemple des relents d'hostilité ethnique ${ }^{7}$ ). Comme j'ai pu le constater en travaillant moi-même ces sensations en suivant ton exemple, l'attention à soi et l'écoute de ses propres ressentis corporels sont déterminants dans la relation à l'autre. Il ne s'agit pas seulement d'un sentiment moral qui nous permettrait de sentir comme en lieu et place de l'autre, par empathie, mais d'une capacité d'appréhension de soi dans la conscience du corps qui accompagne une pratique de la moralité. L'exigence est donc double : elle requiert l'attention aux attentes, aux besoins, aux spécificités de l'autre sans laisser de côté la conscience de soi et de son propre corps.

- De par mon expérience, la conscience du corps est indispensable si l'on veut atteindre un degré de conscience de soi suffisant pour être en accord avec son être profond. Plus la conscience psychocorporelle est fine, plus on est relié à soi, donc en lien avec son intégralité et susceptible d'être à l'écoute de sa corporéité, de ses ressentis et de ses messages corporels, physiologiques et émotionnels. Cette conscience est un vecteur d'anticipation très sensible de ses signaux, à condition d'être vraiment à l'écoute de ses sensations. Ainsi, je sens une maladie " arriver», de même que je me positionne en fonction de ce que je "sens » face à une personne ou dans une situation donnée. La conscience psychocorporelle est une force qui exige une discipline constante. C'est une approche active, holistique et vivante de soi, le socle de toute empathie, me semble-t-il. Sans cette conscience, je pense qu'il est difficile, si ce n'est impossible, de s'inscrire pleinement dans son environnement, d'être par conséquent présent à soi et à l'autre.

- Tu as développé une faculté d'écoute des microperceptions corporelles (digestives, respiratoires, tactiles) que les personnes valides méconnaissent souvent. Dans ton cas, la situation de handicap a rendu nécessaire (et parfois vitale) la conscience du corps, que l'on rencontre entre autres dans le yoga avec l'attention à la respiration, à la posture, à la tension musculaire, à la mobilité, à la survenue des émotions, etc. Cette conscience peut être développée sous l'angle éthique, comme je l'ai dit, et elle consiste en une acuité sensorielle. Mais là où l'individu valide développera plus la sensation articulaire et musculaire, la personne paralysée et capable de sensation sera, par exemple, amenée à se focaliser sur la «sensation de l'os ${ }^{8}$ ou toutes autres microsensations apparemment imperceptibles pour des raisons de confort ou de survie.

- Tout est dit. Du fait de ma fragilité, mes rétractions et mes déformations, toute négligence et la moindre inattention peut coûter très cher et provoquer des souffrances inimaginables. De ce fait, les microperceptions corporelles sont capitales. C'est au mieux une question de bien-être, au pire, une question de vie ou de mort. La réactivité est vitale. Avec cette approche, on comprend que l'être humain est un tout dont on ne peut pas négliger une partie sans en payer tôt ou tard les conséquences. En somme, en la circonstance, nécessité fait loi. Un handicap est une dure école de la vie, une école sans complaisance et sans concession du savoir-vivre et du savoir-être. Ce qui explique nombre de résignations.

— Mais à terme ton expérience personnelle peut enrichir celle des individus «valides », en leur ouvrant de nouvelles perspectives, comme ce fut le cas pour moi. J'ai mieux compris par ton intermédiaire que l'attention au ressenti corporel se distinguait du mouvement et de l'action musculaire. La création d'aptitudes originales et inattendues, comme la conscience du corps chez un homme paralysé, peut contribuer à l'intérêt collectif, surtout lorsque cette attention au ressenti individuel est aussi ouverture à l'autre. Créer ses propres normes, être autrement capable, cela ne signifie pas nécessairement empiéter sur la liberté des autres. Ce qui évite l'objection majeure au développement de l'autonomie individuelle : qu'elle ne soit qu'individuelle, au détriment d'autrui, qu'elle ne soit qu'une revendication personnelle nuisant à la vie en commun. Mais cette possibilité

5 La présence à l'autre est le titre de l'un des livres de Marcel Nuss (Paris, Dunod, 2ème édition, 2008)

6 Philosophe contemporain et praticien de la méthode Feldenkraïs.

${ }^{7}$ Richard SHUSTERMAN, Conscience du corps, Paris, l'Eclat, 2007, p. 42.

${ }^{8}$ En fait cette sensation doit être rapportée au périoste, la couche de tissu qui entoure l'os, celui-ci n'étant pas innervé. 
d'apports réciproques n'existe pas sans une reconnaissance collective de cette autonomisation, sans une voix laissée aux personnes concernées pour demander en retour de leur propre adaptation l'adaptation du corps social. Dans l'exemple de la présence à l'autre, l'autonomie individuelle participe au bien commun. Et elle engage à une réciprocité dans la conscience de soi.

Ton expérience de la dépendance physique totale nous permet de nous comprendre tous comme pourvus d'une autonomie relationnelle, idée que l'on trouve dans les théories du care, montrant qu'aucun d'entre nous n'est autonome seul en société et que nous devons accueillir et assumer cette dépendance.

- Pour moi, le bien-vivre passe par la prise en compte de son prochain. Lorsque l'on vit en situation de dépendance vitale, c'est une évidence. Vivre au détriment d'autrui, dans un individualisme sourd et aveugle, se retourne tôt ou tard contre soi. "Moi-je» est une posture nuisible car contre-productive à terme. Le handicap, s'il est pleinement assumé, intégré, donne une acuité particulière à ce truisme. J'ai besoin de l'autre, c'est évident, mais l'autre aussi a besoin de moi, c'est moins évident à première vue et pourtant... La pleine conscience de cette réalité, de cette vérité, permet la mise en ouvre d'interrelations interactives humanistes et humanisantes, sans lesquelles je ne peux pas prétendre être ou devenir autonome.

- L'autonomie relationnelle dont tu parles ici est plus qu'une indépendance : elle doit être entendue comme la faculté de créer ses propres règles de vie (ses propres normes individuelles, $\mathrm{y}$ compris physiologiques ${ }^{9}$ ). Elle se construit peu à peu en fonction de son milieu et de ses expériences, comme une assise personnelle dans la vie. Cette autonomie, même sur son versant individuel, n'est donc pas strictement fonctionnelle comme on l'entend souvent («autonomie motrice », « autonomie respiratoire »...) : la faculté de se mouvoir n'est qu'un aspect de l'autonomie, assez inutile sans une réelle capacité de choix, sans une autonomie psychique permettant de prendre des décisions et de les assumer. Être autonome signifie construire peu à peu ce dont on est capable, sans se limiter par l'effet d'une intériorisation du regard social sur le handicap par laquelle on se conforme à l'image que l'on reçoit de soi. Or, nous sommes peut-être éminemment humains dans les moments où nous montrons que nous pouvons être plus que ce que nous pensions, ou que ce que l'on imaginait pour nous. La mobilité psychique est une qualité tout aussi éminente dans l'autonomie que les autres formes de mobilité.

- D’une part, plutôt que d'autonomie physique ou respiratoire, je préfère parler d'indépendance ou de dépendance (j'ai une dépendance physique et respiratoire, ce qu'on appelle un plurihandicap), car, sur le plan physique et respiratoire, je n'ai pas créé ma propre norme, elle m'a été imposée par la nature, si j'ose dire; je me suis adapté à cet état singulier et non l'inverse.

D'autre part, s'il est avéré que peu de gens sont autonomes, sauf erreur de ma part c'est encore moins fréquent chez les personnes " handicapées ». Pour devenir vraiment autonome, lorsque l'on a un handicap, a fortiori quand on est en situation de dépendance vitale, il faut d'abord assumer et dépasser le poids, les limites, les contingences et les stigmates inhérents à son handicap. C'est un travail titanesque. D'autant que la majorité des personnes en situation de handicap survivent sous le régime de l'assistanat compassionnel, bénéficiant au mieux d'une " autonomie assistée », subie ou (inconsciemment) choisie. Comment devenir autonome quand, à longueur de temps, on vous assène, d'une manière ou d'une autre, que vous êtes irrémédiablement dépendant, que vous n'avez pas d'avenir, pas d'autre issue possible qu'une solidarité charitable, que vous êtes éduqué dans un environnement fataliste et résigné qui vous empêche ou vous interdit de faire le deuil de vos incapacités rédhibitoires? Car il faut faire le deuil de ses incapacités pour découvrir et développer ses capacités, il faut apprendre à renoncer et cesser de se résigner.

Il me semble nécessaire de préciser que les personnes déficientes intellectuelles ont une réelle mobilité psychique, dans la limite de leurs capacités bien sûr. Pourtant, celle-ci est souvent négligée

\footnotetext{
${ }^{9}$ Georges Canguilhem parle à plusieurs reprises dans ses livres de la possibilité de faire «craquer les normes », même physiologiques, en allant au-delà de ce qu'un corps est censé pouvoir accomplir. Il cite notamment les résultats obtenus par les yoguis : « obtenir un changement du rythme du pouls allant de 50 à 150, une apnée de 15 minutes, une abolition presque totale de la contraction cardiaque, c'est bien faire craquer les normes physiologiques. À moins qu'on choisisse de tenir pour pathologiques de tels résultats » (Georges CANGUILHEM, Le normal et le pathologique, p. 106-107).
} 
ou niée, par méconnaissance, commodité ou laxisme. Ce constat est attristant et révoltant.

- En effet la vie psychique de ces personnes est souvent niée, ou pathologisée comme si elle se réduisait à l'expression de leur atteinte intellectuelle. Or elles aussi ont eu à s'adapter tout au long de leur vie à un environnement humain qui n'était pas fait pour elles, dans lequel elles se sentaient souvent en échec, mais où elles ont pu créer, ou essayer de créer, leurs propres normes de vie.

L'autonomie, comme capacité de création de règles de fonctionnement propres, est une expression de la vie psychique du sujet concerné dans ses spécificités, ce qui interroge également sur l'aide et l'accompagnement: peut-on aider quelqu'un d'autre à trouver une manière d'être, un style d'existence qui ne correspond pas à ce que l'on connaît soi-même ? Comment l'amener à découvrir quelque chose qui pourrait lui être propre mais que les «valides » n'ont pas développé ?

- La réponse est clairement oui. À condition d'être empathique, présent à l'autre sans jugement ni préjugé ou préconçu et dans un réel détachement. En étant sincèrement désintéressé et soucieux du bien-être de la personne accompagnée, donc à l'écoute de sa spécificité et de sa singularité. De ses capacités originales et originelles.

- L'important n'est donc pas de pointer nos limitations mais de percevoir comment chacun d'entre nous peut devenir «autrement capable» de mener sa vie, voire de s'accomplir. Ce qualificatif d'autrement capable peut être attribué à tout un chacun, car nous restons tous, tout au long de notre vie, capables de créer de la nouveauté. C'est cela que l'on peut nommer autonomie au sens propre (la construction de ses normes propres).

Inversement, la notion de dépendance peut s'appliquer à des situations beaucoup plus variées que la dépendance physique. Bien des personnes considérées comme valides sont en réalité dans des états de grande dépendance psychique et affective. Nombre d'entre elles, dont je suis sans doute, sont dépendantes de la présence de leur conjoint, dépendantes de leur bien-être matériel, dépendantes de leurs plaisirs, de leur apparence... Certaines personnes que l'on juge ordinaires n'arrivent pas à envisager le changement : la moindre modification dans les routines (les rituels ?) du quotidien entraîne des doses d'angoisse massives. J'ai connu des personnes qui ne dorment que quelques heures par nuit, aux prises avec des troubles obsessionnels-compulsifs qu'elles dissimulent tant bien que mal, d'autres pour lesquelles tout événement, même positif, paraît insurmontable, comme le fait qu'un enfant prenne désormais son indépendance et vive sa vie loin de ses parents... Je choisis volontairement des exemples de la vie courante pour montrer la banalité des dépendances psychiques, la prégnance des sentiements de culpabilité ou de mésestime de soi.

- De par mon expérience, j'ai le sentiment que la dépendance psychique est plus handicapante qu'une dépendance physique, aussi importante soit-elle. En effet, même si je suis prisonnier de mon corps, je suis psychiquement libre et autonome, souvent bien plus que les gens qui m'entourent, car je vis l'instant présent sans être dépendant des autres autrement que physiquement, pleinement conscient de mon intégrité et de mon intégralité. Je suis un tout autonome et profondément soucieux de l'autonomie d'autrui, par conséquent de ses limites. L'autre ne m'appartient pas, pas plus que je ne lui appartiens. Mais, pour attendre ce niveau d'autonomie, il a fallu des années de travail, de déconvenues et d'errements. Pas plus qu'on ne naît handicapé, on ne naît autonome, on le devient à force d'apprentissage.

De plus, il ne faut pas négliger l'impact des dépendances religieuses et socioculturelles qui agissent ou interagissent de façon très puissante et prégnante sur la dépendance psychoaffective. Nous sommes le fruit et le résultat d'une culture, avant de devenir autonome en nous démarquant de cette emprise culturelle parfois insidieuse.

- L'intériorisation des normes sociales, groupales et familiales est telle qu'en effet nous ne recevons pas la liberté : nous la construisons peu à peu. Une liberté ne s'achète pas, ne se prend pas à autrui pour en profiter à sa place. Elle se développe contre les difficultés, que ce soit à l'échelle sociale ou individuelle ${ }^{10}$.

En ce qui te concerne, on est en droit de se demander comment tu es parvenu à contourner autant de difficultés dans ta propre vie : comment penses-tu être devenu indépendant de tes angoisses et

\footnotetext{
${ }^{10}$ Jean-Paul Sartre évoque cette prise de liberté (au sens d'une prise de risque) dans « La République du Silence », in Situations III, Paris 1964, p. 11-14.
} 
pensées négatives?

- Par un travail permanent sur moi-même, une remise en question régulière, un questionnement incessant, suscités par une attention à mes perceptions sensorielles et émotionnelles et une écoute sans complaisance des retours et des interpellations objectives ou subjectives d'autrui. Je me suis toujours regardé en face ; même s'il m'est arrivé de me voiler la face ou d'en avoir la tentation, cela n'a jamais duré très longtemps, parce que la réalité et la vérité m'ont toujours ouvert les yeux et le cour, parfois de façon cuisante. La vie, la vraie, est sans concession, sans faux-semblant.

- Je suppose que cette aptitude peut être envisagée sous l'angle du rapport au temps et de la forme que prend la mémoire du passé. Par exemple: tes souvenirs sont-ils anecdotiques ou synthétiques? Pour ma part, ils sont souvent anecdotiques, et j'ai la fâcheuse tendance à ne retenir d'une belle journée qu'un moment désagréable qui a perturbé l'équilibre d'ensemble. J'ajoute que je n'envisage pas mes souvenirs de la même manière en fonction de mon état du moment : loin d'être seulement une évocation de ce qui a été, ils sont souvent la marque de ce que je pense et de ce que je sens actuellement.

- Mes souvenirs sont lointains; de ce fait, écrire mon autobiographie ${ }^{11} \mathrm{~m}$ 'a souvent été pénible. J'ai la capacité - que j'estime être une qualité très appréciable - de tourner la page très vite, de ne jamais avoir de regrets ni de nostalgie. Je ne regarde pas en arrière, je vais de l'avant. Le passé est passé, ce qui a été ne sera plus, seul le présent et le futur m'importent. Il faut vivre en s'adaptant au mieux aux circonstances.

- Quand on lit cette autobiographie, se dit que les situations terriblement difficiles que tu as eu à affronter ont dû te laisser des souvenirs traumatiques, du type de ceux qui hantent le psychisme sous forme de reviviscences. La répétition d'une situation pénible, même peu intense, comme des soins inappropriés, peut produire par cumul un impact traumatique. Dans de telles circonstances, comme l'écrit René Roussillon, «la blessure psychique n'a jamais le temps de cicatriser, elle est sans cesse réouverte, devient de plus en plus béante avec le temps et la répétition ${ }^{12}$. Quel est ton point de vue sur ce que l'on est en droit d'appeler des traumatismes cumulatifs ?

- Je ne m'appesantis pas sur mes souvenirs, encore moins lorsqu'ils sont pénibles. À quoi bon? Je n'y pense pas spontanément; s'ils ressurgissent c'est à l'occasion d'une interpellation ou de la question d'un tiers. J'ai "subi » de nombreux impacts traumatiques, j'ai des blessures psychiques, mais je les "soigne " en m'inscrivant dans le présent : qu'est-ce que je fais de cette souffrance aujourd'hui, avec qui je suis aujourd'hui? On ne peut pas changer ce qui a été mais on peut influer sur ce qui sera. C'est une sagesse élémentaire que m'ont inculqué le temps et l'expérience. Tout le reste n'est que perte d'énergie et de sens.

- A propos de cette vision de ce qui sera, quel est ton rapport à l'avenir, à la menace de la mort qui paralyse tant d'êtres humains?

- Je vis avec la mort depuis ma petite enfance. Je l'ai donc apprivoisée, ou elle m'a adopté. Pour moi, la mort fait partie de la vie, ce n'est pas une fin en soi, c'est le commencement d'autre chose. De quoi ? Je ne sais pas. Et, à vrai dire, ce n'est pas mon souci. À chacun sa réponse, en fonction de ses croyances et de ses angoisses. En ce qui me concerne, la vie n'est pas une question de quantité mais de qualité. Ça ne sert à rien de s'arc-bouter contre l'inéluctable, c'est une perte de temps ; il faut surfer sur la vie afin de la savourer au quotidien.

- Je te rejoins là-dessus, et il existe des pratiques et activités qui permettent de développer en soi l'appétit de vivre et d'exister, comme la méditation, l'intention éthique envers autrui ou l'attention au plaisir. Ce sont de bons moyens pour lutter contre les pensées obsédantes, ces pensées qui ne laissent pas de repos, parce qu'elles tournent et retournent dans l'esprit sans jamais de pause. En fait, ce ne sont pas seulement des pensées : ce sont des pensées-affects, chargées affectivement. Elles sont chargées de représenter ce qui fait leur charge émotionnelle. Ainsi la pensée-affect de la mort peut aussi véhiculer des sensations d'impuissance, de limitation, de honte que l'analyse du

\footnotetext{
${ }^{11}$ Marcel NUSS, En dépit du bon sens : autobiographie d'un têtard à tuba, Paris, éditions de l'Éveil, 2015.

${ }^{12}$ René ROUSSILLON, « Jalons et repères de la théorie psychanalytique du traumatisme psychique »

(https://reneroussillon.files.wordpress.com/2014/08/th-psy-trauma-01.pdf)
} 
concept de mort ne suffira pas à les épuiser.

- Moi aussi je fais l'expérience des pensées-affects obsédantes. De moins en moins souvent, fort heureusement, mais je n'en suis pas exempt pour autant. La dernière fois que cela m'est arrivé, c'est en décembre 2015. Elles étaient nourries par un profond sentiment de trahison et d'injustice, à l'origine duquel il y avait plus particulièrement un de mes accompagnants, que j'ai licencié dans la foulée en raison d'un comportement mesquin, irrespectueux et injuste. En fait, la déflagration de ces pensées-affects était proportionnelle à l'attachement que j'éprouvais alors pour cette personne qui avait travaillé à mes côtés pendant quatre ans. Je me suis mis dans un tel état que ça m'a littéralement rendu malade et que j'ai eu des problèmes cardiaques. Car j'étais incapable d'arrêter ces pensées, incapable de maîtriser mes émotions.

- Je comprends bien cette proportionnalité entre l'attachement et la souffrance de la perte... J'en ai subi les conséquences moi aussi il y a des années... Pour les situations moins graves, j'ai appris à distinguer les pensées négatives et l'état physique qui les suscitait en moi : au lieu d'être pris dans le cercle de ces pensées dépréciatives, je peux désormais les mettre à distance et me rapprocher du présent, mais dans une moindre mesure que toi.

Ce que tu indiquais n'est pas si loin de ce que prônaient les auteurs antiques : apprendre à profiter du plaisir pour les épicuriens, apprendre à modifier ses représentations pour les stoïciens : discerner ce qui dépend de nous (sur quoi nous avons prise) et ce qui n'en dépend pas (qui ne doit pas nous toucher).

La fréquentation d'autrui peut permettre ce développement de soi. Nous nous découvrons souvent nous-mêmes par ce que les autres nous révèlent, soit que nous nous identifions à autrui (comme dans l'enfance à des adultes appréciés), soit que nous finissions par accepter le regard bienveillant que d'autres portent sur nous comme chargé d'une part de vérité. L'exemple le plus frappant à ce propos est la relation amoureuse : l'amour n'est-il pas une manière de trouver sa vérité en l'autre?

Pour autant, rien ne peut nous permettre d'affirmer que nous connaissions autrui, si forte soit la relation qui nous unit. Tous mes efforts dans la relation de couple se sont portés sur la capacité à identifier la manière dont ma femme éprouvait ce que l'on nomme «la vie partagée », à me rapprocher de son monde intérieur (parce qu'elle me le permet), et cette expérience singulière est souvent tout aussi difficile à saisir que celles de personnes que je rencontre dans mes travaux sur le handicap... L'expérience subjective est largement ineffable. Mais il n'est pas interdit de tenter de contourner cette difficulté en essayant de décrire sa propre expérience au plus près de l'autre...

$\mathrm{Au}$ sein d'un couple, l'un et l'autre peuvent être très heureux pour des raisons entièrement différentes, ce qui ne pose aucun problème... mais qu'en est-il lorsque l'on se sent très mal pour des raisons que l'on n'arrive plus à cerner, faute d'avoir appris à se parler ? Cela ne fait-il pas partie de la manière dont tu conçois toi aussi la séduction et la relation de couple ?

- Si, entièrement. Pour moi l'amour, et particulièrement la vie de couple, c'est une alchimie dont la qualité dépend surtout de la capacité de celui-ci à dialoguer de tout et sans tabou. Ce qui, pour la majorité des couples, d'après mon expérience est plus vite dit que fait. Je n'ai pas la prétention de connaître l'autre, l'aimée, celle qui a fait le choix de partager ma vie un temps durant, mais je peux m'en approcher au maximum en dialoguant avec elle. En fait, ma vie et mon handicap m'ont appris que les non-dits tuent. Avec ma femme, notre rituel immuable est de dialoguer à bâtons rompus tous les soirs, au lit, jusqu'à une heure ou deux heures du matin. Ça nous est indispensable. C'est un des ciments de notre couple, donc de notre amour. De plus, nous sommes d'accord que l'autre ne nous appartient pas, et qu'un couple est l'union de deux solitudes qui se rejoignent car elles se sont reconnues au moment de leur rencontre.

- Sans mon épouse, il y aurait quantité d'expériences et de découvertes que je n'aurais pas faites, et je pense que cela est loin de se terminer... C'est pour moi un complément remarquable à ce que je peux apprendre théoriquement par les textes, ou un complément aux travaux sur le handicap que nous menons l'un et l'autre. Mais encore faut-il compter, comme tu sais si bien le faire, sur la capacité à repérer les états émotionnels des autres...

- Oui, je ressens les autres, c'est un ressenti physique indicible. C'est de l'ordre de l'instinctif 
ou de l'intuitif, ou des deux. J'ai comme un "radar aurique » qui se met en route. Je le ressens soit dans la poitrine, soit dans le plexus. Dans le second cas, en général, cela m'annonce que la personne est négative, donc que je dois me protéger en gardant un certain recul et en me mettant dans une bulle blanche. Dans le premier cas, je dois parfois me protéger de moi-même, d'un élan spontané vers la personne trop impulsif et affectif; je m'emballe trop vite, en somme.

Je pense que nous avons tous un "radar» en nous mais que peu savent s'en servir, par facilité, indifférence ou manque de confiance en ses capacités. L'humain se sous-estime énormément. Hélas pour l'humanité. Mais cela viendra peut-être quand sa survie dépendra de nouveau de son acuité sensorielle et émotionnelle.

\section{Bibliographie}

ANCET P. (dir.). Le Corps vécu chez la personne âgée et la personne handicapée, Paris, Dunod, 2010.

ANCET P. et TOUBERT-DUFFORT D. (dir.). dossier «Corps, identité, handicap », Nouvelle revue de l'adaptation et de la scolarisation, éditions de l'INS-HEA, n ${ }^{\circ}$, juillet 2010, p. 2-162.

CANGUILHEM G. (1943, 2ème éd. augmentée 1966). Le Normal et le pathologique, Paris, PUF, 1995

NUSS M. (2011). La Présence à l'autre, Accompagner les personnes en situation de dépendance, $3^{\mathrm{e}}$ édition, Paris, Dunod.

NUSS M. et Cohier-Rahban V. (2011). L'identité de la personne « handicapée », Paris, Dunod.

NUSS M. et ANCET P. (2012). Dialogue sur l'altérité et le "handicap » : ressemblances dans la différence, préface de Serge Tisseron, Paris, Dunod.

NUSS M. (2012). Je veux faire l'amour, Paris, Autrement.

NUSS M. (2015). En dépit du bon sens : autobiographie d'un têtard à tuba, préface de Michel Onfray, L'Éveil Citoyen.

NUSS M. (2016). Oser accompagner avec empathie, préface d'André Comte-Sponville, Paris, Dunod.

SHUSTERMAN R. (2007). Conscience du corps, Paris, l'Eclat. 